\title{
Three-Dimensional Printed Patient Models for Complex Pediatric Spinal Surgery
}

\author{
Jeffrey D. Coote, MD, ${ }^{1}$ Theresa Nguyen, MBBS, ${ }^{1}$ Kaitlyn Tholen, ${ }^{2}$ Caleb Stewart, BS, ${ }^{1}$ Elizabeth Verter, MBBS, ${ }^{1}$ \\ John McGee, BSE, ${ }^{3}$ Paul Celestre, MD, ${ }^{1,4}$ Korak Sarkar, MD ${ }^{1,3,5}$ \\ ${ }^{1}$ The University of Queensland Faculty of Medicine, Ochsner Clinical School, New Orleans, LA ${ }^{2}$ Tulane University School of Science and \\ Engineering, New Orleans, LA ${ }^{3}$ Ochsner Medical 3D Laboratory, New Orleans, LA ${ }^{4}$ Department of Orthopedic Surgery, Ochsner Clinic \\ Foundation, New Orleans, LA ${ }^{5}$ Department of Neurology, Ochsner Clinic Foundation, New Orleans, LA
}

Background: Pediatric spinal deformity surgeries are challenging operations that require considerable expertise and resources. The unique anatomy and rarity of these cases present challenges in surgical training and preparation. We present a case series illustrating how 3-dimensional (3-D) printed models were used in preoperative planning for 3 cases of pediatric spinal deformity surgery.

Case Series: Patient 1 was a 6-year-old male with scoliosis secondary to an L3 hemivertebra and severe congenital heart disease who underwent excision of the L3 hemivertebra and L2-L4 spinal fusion. Patient 2 was an 11-year-old male with an L2 hemivertebra and lumbar kyphosis who underwent excision of the L2 hemivertebra and T12-L4 spinal fusion. Patient 3 was a 6-year-old female with Down syndrome who presented with atlantoaxial instability and acute lymphoblastic leukemia. She underwent occipitalcervical spinal fusion and decompression. Prior to surgery, 3-D printed models of the patients' spines were created based on computed tomography (CT) imaging.

Conclusion: The anatomic complexity and risk of devastating neurologic consequences in spine surgery call for careful preparations. 3-D models enable more efficient and precise surgical planning compared to the use of 2-dimensional CT/magnetic resonance images. The 3-D models also make it easier to visualize patient anatomy, allowing patients and their families who lack medical training to interpret and understand cross-sectional anatomy, which in our experience, enhanced the consultations.

Keywords: Imaging-three dimensional, pediatrics, orthopedics, scoliosis, spinal fusion, spine

Address correspondence to Korak Sarkar, MD, Department of Neurology, Ochsner Clinic Foundation, 1514 Jefferson Hwy., New Orleans, LA 70121. Tel: (504) 897-5854. Email: korak.sarkar@ochsner.org

\section{INTRODUCTION}

Introduced in the 1980s, additive manufacturing-or 3dimensional (3-D) printing - has become an important tool in medicine with multiple applications, ranging from implant and tissue design to medical research and training. ${ }^{1}$ Additive manufacturing has rapidly emerged as an essential tool for operative planning and training in a variety of fields including neurosurgery, orthopedics, otolaryngology, and craniomaxillofacial and cardiac surgery. ${ }^{2-7}$ Additive manufacturing allows surgeons to personalize their surgical planning to each patient and helps improve patient education, comprehension, and satisfaction. ${ }^{8}$

A principal use of additive manufacturing is the creation of 3-D models for preprocedural planning; this application is particularly relevant in complex spinal deformity cases involving anatomic anomalies that are rarely encountered in surgical training or practice. For example, surgery for congenital pediatric spinal deformity is uncommon, with $1-10 / 100,000$ children having a congenital spinal anomaly requiring surgical intervention. ${ }^{9}$ Because of the infrequent occurrence of these cases, gaining and maintaining surgical proficiency can be difficult. Moreover, while 2-dimensional (2-D) imaging modalities have been the standard imaging modality used for these deformities, the spine is a 3-D object with complex anatomic structures and relationships that are not completely appreciated in 2 dimensions. 3-D printing of spine models allows surgeons to more completely understand the anatomy of complex spinal deformities; facilitates better preparation for an operative intervention; and potentially minimizes operative time, blood loss, and the overall risk of neurologic injury. ${ }^{10,11}$

We report our experience with additive manufacturing, specifically highlighting 3 complex cases in which our 3-D models were used for preoperative planning and for patient education.

\section{IMAGING PROCEDURE}

The Ochsner Medical 3D Laboratory acquired the Digital Imaging and Communications in Medicine (DICOM) image files of patients' computed tomography (CT) spine scans 
in conjunction with the Ochsner Radiology and Pediatric Orthopedic Surgery departments. All investigations were considered part of normal clinical care, and the use of patients' radiographic imaging for production of 3-D printed models was approved by the institutional review board. We used Mimics inPrint (Materialise) to segment regions of interest and created 3-D spine renderings. Models were printed by offsite partner Entrescan with a variety of printers (Formlabs 2S [Formlabs, Inc.] and ProJet 660 [3D Systems]) and materials (eg, photoreactive resins and gypsum powder).

\section{CASE SERIES}

The primary surgeon for each case used the 3-D printed models to help determine his surgical approach (measured angles of curvature, degree of rotation of the spine). He also used the models for patient and family education to describe the patient's anatomic abnormalities and the surgical approach he would use to correct the abnormalities.

\section{Patient 1}

A 6-year-old male with a history of hypoplastic left heart syndrome status post fenestrated Fontan, heterotaxy syndrome, primary ciliary dysfunction, left-sided bronchiectasis, growth delay, and congenital scoliosis secondary to an L3 hemivertebra presented for evaluation of left leg weakness. At the time of evaluation, he had seen multiple surgeons and required orthotics for ambulation. Additionally, the patient had developed a right lower extremity radiculopathy resulting in significant right lower extremity night pain. Radiographic imaging demonstrated an apex left lumbar scoliosis of 40 degrees centered at the L3 hemivertebra (Figure 1), and the patient was scheduled for surgical treatment of his scoliosis with a planned hemivertebra excision. A 3-D model of the patient's spinal column was created for preprocedural planning (Figure 2).

The patient underwent L2 laminectomy, excision of the L3 hemivertebra, and posterior spinal fusion of L2-L4 with pedicle screw and rod placement. Operative time was 2 hours, 32 minutes; estimated blood loss (EBL) was $150 \mathrm{~mL}$. The procedure was complicated by a cerebrospinal fluid leak involving the left L3 nerve root axilla that was repaired using Prolene sutures (Ethicon). The patient had no significant postoperative complications. At follow-up, his pain had resolved, and x-rays demonstrated minimal residual lumbar scoliosis.

\section{Patient 2}

A 11-year-old male with Hurler syndrome, global developmental delay, sickle cell trait, chronic respiratory infections, obstructive sleep apnea, and multiple cardiac anomalies presented for evaluation of an L2 hemivertebra with resultant congenital kyphosis. He complained of new-onset back pain and symptoms consistent with sciatica. Radiographic imaging demonstrated a thoracolumbar kyphosis of 55 degrees (increased from a previously stable 50-degree kyphosis). Figures 3 and 4 are digital renderings of the patient's congenital kyphosis, L2 hemivertebra, and congenital scoliosis. A 3-D model of the patient's spine (not shown) was created for preprocedural planning.

The patient underwent an anterior L2 hemivertebra excision with posterior spinal fusion of T12-L4 in an effort to cor-

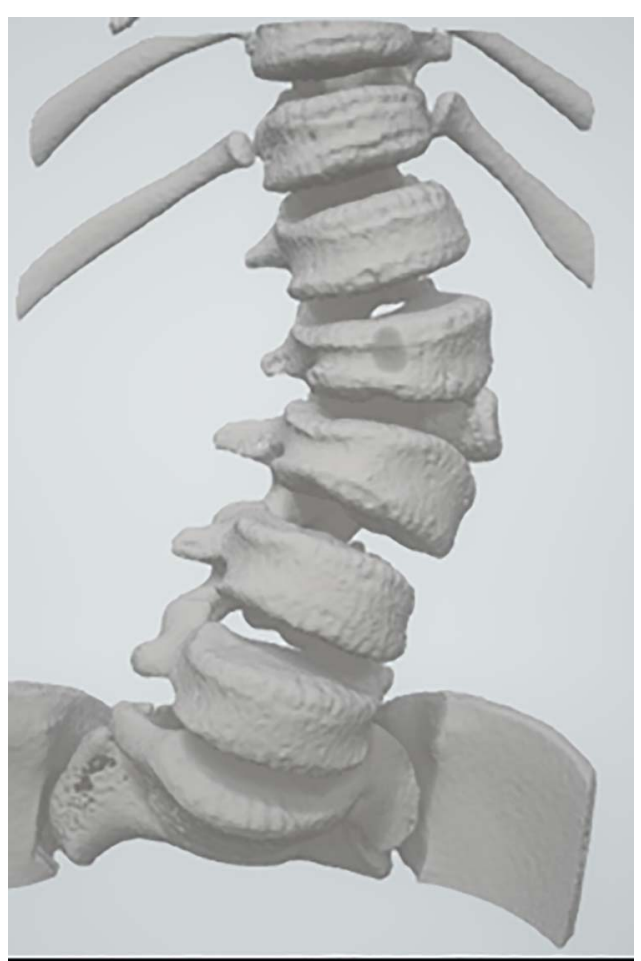

Figure 1. Patient 1: Digital rendering of patient's lumbar scoliosis, anterior view.

rect his progressive thoracolumbar kyphosis and complaints of worsening neurologic symptoms and back pain. The procedure lasted 5 hours, 25 minutes; EBL was $150 \mathrm{~mL}$; and no intraoperative complications occurred. The patient's postoperative course was benign, and no complications were

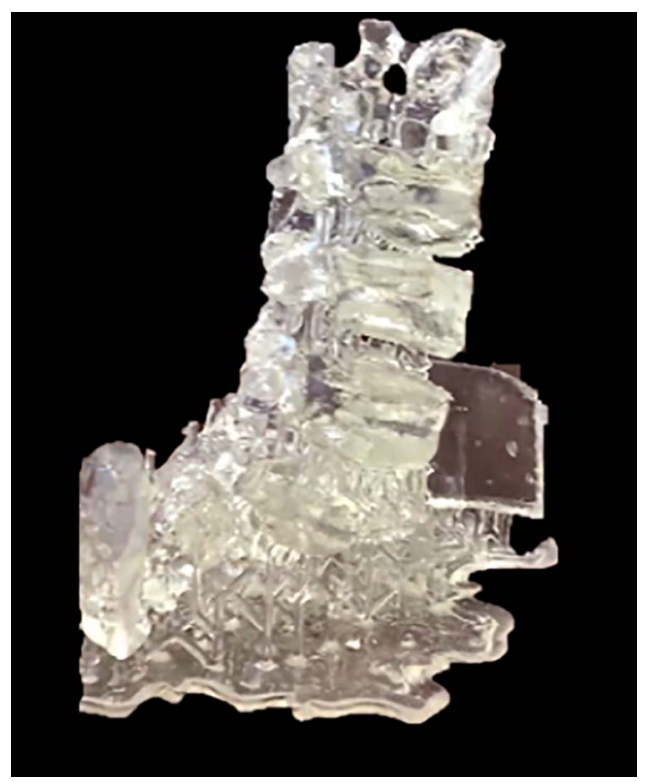

Figure 2. Patient 1: Three-dimensional printed patient-specific model of congenital scoliosis secondary to an L3 hemivertebra used for preprocedural planning. 


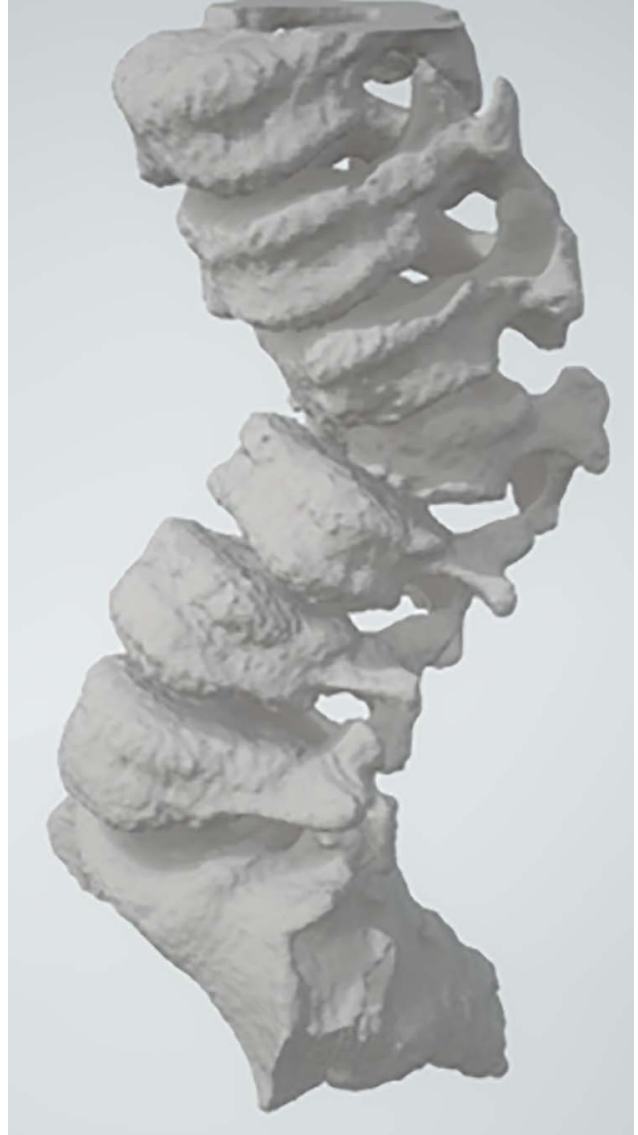

Figure 3. Patient 2: Digital rendering of patient's congenital kyphosis and L2 hemivertebra, lateral view.

identified at 3-month follow-up. He returned to school, and his only new restriction was the inability to participate in physical education class.

\section{Patient 3}

A 6 -year-old female with trisomy 21 , acute lymphoblastic leukemia, and hypothyroidism presented with progressive myelopathic symptoms, including broad-based gait, frequent falls, and difficulty holding objects. Radiographic imaging demonstrated atlantoaxial instability and cervical stenosis of the spine resulting in progressive cervical myelopathy. A 3-D model of her CT images was developed and printed for preoperative planning, procedural consent, and patient education. Figures 5 and 6 present 2 views of the model.

The patient underwent occiput to C5 posterior spinal fusion with rod placement and $\mathrm{C} 1$ laminectomy and foramen magnum decompression for decompression of the spinal cord. The procedure lasted 2 hours, 46 minutes; approximately $50 \mathrm{~mL}$ of blood was lost; and no intraoperative complications occurred. At the patient's 1-month follow-up, her mother noted improvement in the patient's ability to use her right hand. Three months after her procedure, the patient was walking again, although her mother noted some residual right upper and lower extremity weakness.

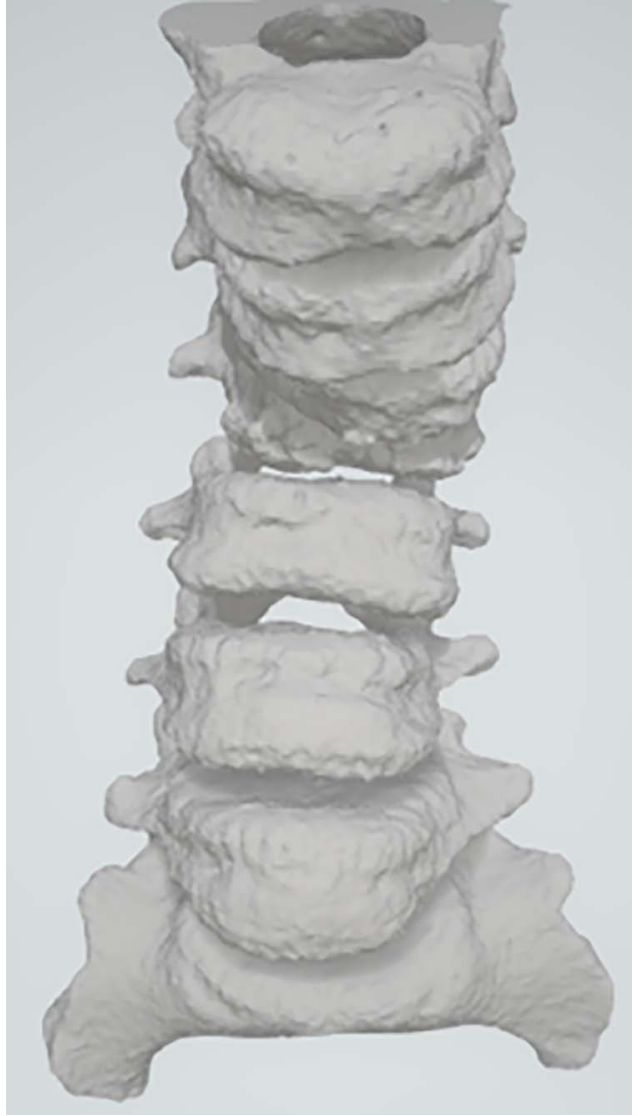

Figure 4. Patient 2: Digital rendering of patient's congenital scoliosis, anterior view.

\section{DISCUSSION}

3-D imaging has become an integral part of the surgical planning process at our institution because it facilitates a more intuitive understanding of anatomy compared to the 2-D images created by CT and magnetic resonance imaging. Surgeons who have implemented 3-D printed models as a part of their preprocedural planning report improved patient outcomes, more complete correction of the deformities being addressed, and decreased operating room time. ${ }^{11-15} 3-\mathrm{D}$ models also allow surgeons to show patients their pathology in a tangible, intuitive way. Consequently, the use of 3-D models in preprocedural planning has been associated with improvement in patient informed consent and in patient/family member perception of proposed procedures. $^{13,14}$ In our study, 2 of the 3 patients' families reported that the 3-D model helped them better understand the anatomic abnormalities and how the proposed surgical approach would attempt to correct the abnormalities.

Surgeons report that these models accurately depict patient anatomy, ${ }^{1,7}$ although more research must be done to quantitatively assess the accuracy of 3-D models compared to PACS (picture archiving and communication system) images and true anatomy.

The 3-D models are relatively inexpensive (the average cost of materials is $\$ 447.50$ ) and can be printed within a few days of completion of the patient's CT scan. Although printing 3-D prototypes provides both the surgeon and the patient with a physical model of the patient's unique anatomic 


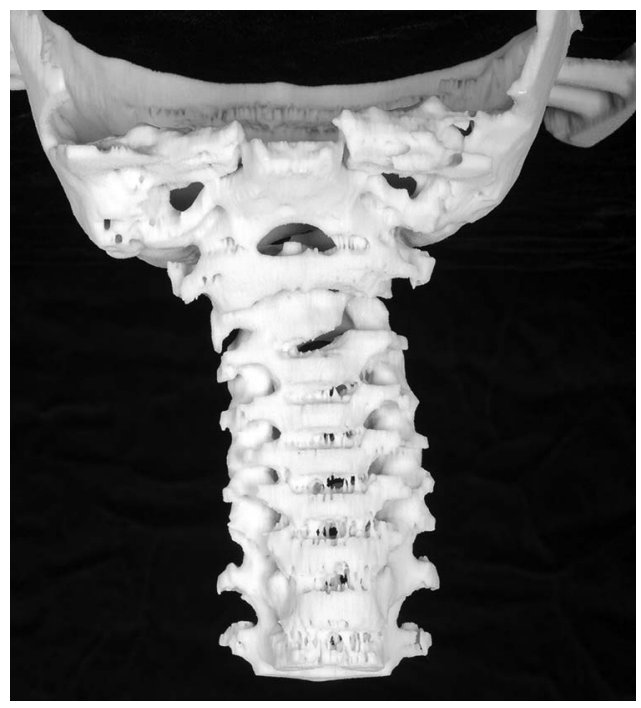

Figure 5. Patient 3: Three-dimensional printed patient-specific model of atlantoaxial instability, coronal view.

makeup, advances in virtual reality (VR) models offer the same 3-D interpretation as the printed counterparts while eliminating the time and cost required to create a physical 3-D model. For this reason, we believe VR has great potential as a complementary tool with faster turnaround than 3-D printing and minimal material costs.

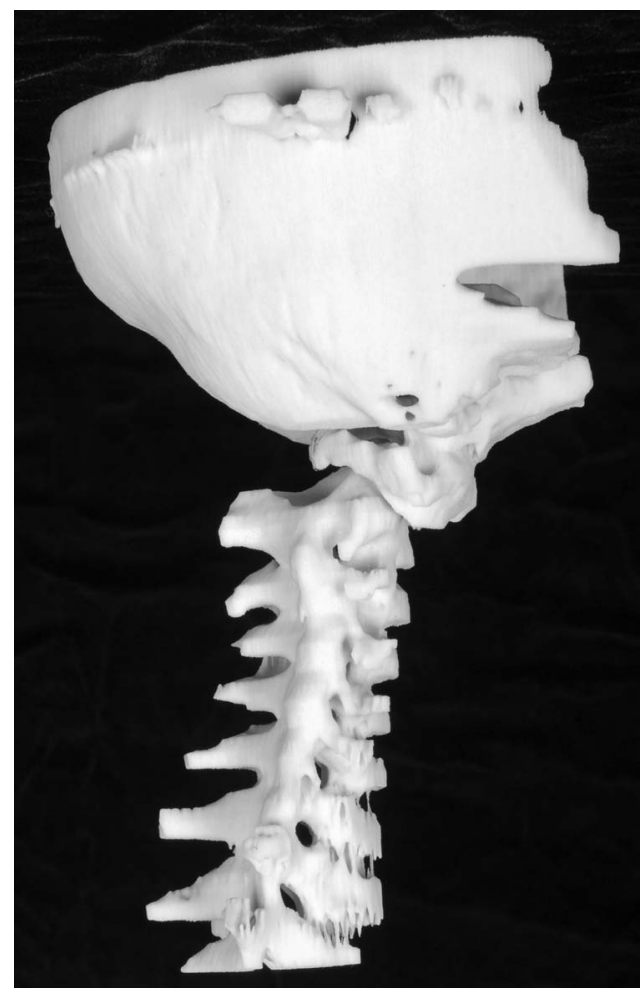

Figure 6. Patient 3: Three-dimensional printed patient-specific model of atlantoaxial instability, sagittal view.
This case series has limitations. One notable limitation is that the models in these cases only depict the bony structures. For these cases, the surgeon requested that bone only be printed to provide a detailed image of the bony abnormalities. However, when planning these surgeries, understanding the relationship of the bony abnormalities to other tissues (ie, neural tissue) is important, as damage to these tissues can result in serious complications. Further, while our experience supports the many benefits of 3-D printing in surgical planning, further research is needed to determine the efficacy of this modality. For example, studies have shown that use of 3-D models in preoperative planning helps decrease time in the operating room; however, the extent by which these models alone decrease operating time is unclear. ${ }^{13,15}$ The next steps to better understand the utility of 3-D printing in surgical planning include assessing the impact on operating room time, intraoperative complications, and postoperative outcomes.

Although the use of additive manufacturing in medicine is still in its infancy, this technology offers the possibility of improving healthcare with its ability to rapidly create customized models. ${ }^{16}$

\section{CONCLUSION}

3-D models can not only improve preoperative planning and provide opportunities to rehearse for complex procedures, but they can also improve consultations with patients by providing easily grasped 3-D renderings of difficult-tointerpret 2-D radiographic images. In our experience, the models enhanced patient understanding and satisfaction. 3$D$ printed patient-specific models have great potential in surgical planning and patient education, but further research is needed to fully explore the efficacy of these tools in complex surgery.

\section{ACKNOWLEDGMENTS}

We would like to thank Entrescan of New Orleans, LA for assistance with printing the 3-dimensional models. The authors have no financial or proprietary interest in the subject matter of this article.

\section{REFERENCES}

1. Rengier F, Mehndiratta A, von Tengg-Kobligk H, et al. 3-D printing based on imaging data: review of medical applications. Int J Comput Assist Radiol Surg. 2010 Jul;5(4):335-341. doi: 10.1007/s11548-010-0476-x.

2. Giovinco NA, Dunn SP, Dowling L, et al. A novel combination of printed 3-dimensional anatomic templates and computer-assisted surgical simulation for virtual preoperative planning in Charcot foot reconstruction. J Foot Ankle Surg. 2012 May-Jun;51(3):387-393. doi: 10.1053/j.jfas.2012.01.014.

3. Levine JP, Patel A, Saadeh PB, Hirsch DL. Computer-aided design and manufacturing in craniomaxillofacial surgery: the new state of the art. J Craniofac Surg. 2012 Jan;23(1):288-293. doi: 10.1097/scs.0b013e318241ba92.

4. Rehder R, Abd-El-Barr M, Hooten $K$, Weinstock P, Madsen JR, Cohen AR. The role of simulation in neurosurgery. Childs Nerv Syst. 2016 Jan;32(1):43-54. doi: 10.1007/s00381-015-2923-z.

5. Schmauss D, Gerber N, Sodian R. Three-dimensional printing of models for surgical planning in patients with primary cardiac tumors. J Thorac Cardiovasc Surg. 2013 May;145(5):1407-1408. doi: 10.1016/j.jtcvs.2012.12.030. 
6. Spottiswoode BS, van den Heever DJ, Chang Y, et al. Preoperative three-dimensional model creation of magnetic resonance brain images as a tool to assist neurosurgical planning. Stereotact Funct Neurosurg. 2013;91(3):162-169. doi: $10.1159 / 000345264$.

7. Waran V, Menon R, Pancharatnam D, et al. The creation and verification of cranial models using three-dimensional rapid prototyping technology in field of transnasal sphenoid endoscopy. Am J Rhinol Allergy. 2012;26(5):132-136. doi: 10.2500/ajra.2012.26.3808.

8. Bernhard JC, Isotani S, Matsugasumi T, et al. Personalized 3-D printed model of kidney and tumor anatomy: a useful tool for patient education. World J Urol. 2016 Mar;34(3):337-345. doi: 10.1007/s00345-015-1632-2.

9. Boachie-Adjei $O$, Cunningham ME. Revision spine surgery in the growing child. In: Akbarnia BA, Yazici M, Thompson GH, eds. The Growing Spine. Heidelberg, Germany: Springer; 2011:487-497. doi: 10.1007/978-3-540-85207-0_38.

10. Newton PO, Fujimori T, Doan J, Reighard FG, Bastrom TP, Misaghi A. Defining the "three-dimensional sagittal plane" in thoracic adolescent idiopathic scoliosis. J Bone Joint Surg Am. 2015 Oct 21;97(20):1694-1701. doi: 10.2106/jbjs.o.00148.
11. Diab M, Smith AR, Kuklo TR; Spinal Deformity Study Group. Neural complications in the surgical treatment of adolescent idiopathic scoliosis. Spine (Phila Pa 1976). 2007 Nov 15;32(24):2759-2763. doi: 10.1097/brs.0b013e31815a5970.

12. Lykissas MG, Crawford AH, Jain VV. Complications of surgical treatment of pediatric spinal deformities. Orthop Clin of North Am. 2013 Jul;44(3):357-370, ix. doi: 10.1016/j.ocl.2013.03.007.

13. D'Urso PS, Barker TM, Earwaker WJ, et al. Stereolithographic biomodelling in cranio-maxillofacial surgery: a prospective trial. J Craniomaxillofac Surg. 1999 Feb;27(1):30-37. doi: 10.1016/s1010-5182(99)80007-9.

14. Izatt MT, Thorpe PL, Thompson RG, et al. The use of physical biomodelling in complex spinal surgery. Eur Spine J. 2007 Sep;16(9):1507-1518. doi: 10.1007/s00586-006-0289-3.

15. Cherkasskiy L, Caffrey JP, Szewczyk AF, et al. Patient-specific 3-D models aid planning for triplane proximal femoral osteotomy in slipped capital femoral epiphysis. J Child Orthop. 2017 Apr;1 1(2):147-153. doi: 10.1302/1863-2548-11-170277.

16. Wilcox B, Mobbs RJ, Wu AM, Phan K. Systematic review of 3-D printing in spinal surgery: the current state of play. J Spine Surg. 2017 Sep;3(3):433-443. doi: 10.21037/jss.2017.09.01.

This article meets the Accreditation Council for Graduate Medical Education and the American Board of Medical Specialties Maintenance of Certification competencies for Patient Care, Medical Knowledge, and Practice-Based Learning and Improvement. 